\title{
Afetos positivos e negativos em professores de diferentes níveis de ensino
}

\author{
Ana Paula Porto Noronha \\ Universidade São Francisco - SP \\ Mariana Palladino Delforno \\ Universidade São Francisco - SP \\ Lariana Paula Pinto \\ Universidade São Francisco - SP
}

\begin{abstract}
Resumo
O presente estudo objetivou comparar afetos positivos e negativos de professores dos diversos níveis de ensino. Participaram 101 professores, com idade média de 39,3 anos (DP = 9,8), sendo 83,2\% ( $n=84)$ do sexo feminino. Utilizou-se a Escala de Afetos Zanon- EAZ. Os professores foram cooptados no próprio local de trabalho, e as aplicações ocorreram tanto individual quanto coletivamente. Para efetivação das análises recorreu-se à ANOVA, por analisar perfis de medidas repetidas. Como um dos resultados mais relevantes, observou-se que os afetos positivos estiveram mais presentes na amostra em questão. Também foi verificado que os educadores de creche apresentaram menos afetos positivos que os demais, fato que pode ter sido influenciado por singularidades percebidas pelos participantes como, por exemplo, as relacionadas à remuneração, à carga horária e ao plano de carreira. Não foram encontradas diferenças quanto ao sexo, e à faixa etária. Observou-se a necessidade de desenvolver mais estudos sobre esta temática.
\end{abstract}

Palavras-chave: Psicologia Positiva, afeição, professores.

\section{Positive and negative affects of teachers from different educational levels}

\begin{abstract}
In this study we aim at comparing positive and negative affects of teachers from different educational levels. 101 teachers participated in this study, being $83.2 \%(n=84)$ female with an average age of 39.3 years $(S D=9.8)$. The instrument used was the Escala de Afetos Zanon - EAZ. Teachers were co-opted in the workplace, and the applications occurred both individually and collectively. In order to carry out the analysis, we used ANOVA method, by analyzing profiles of repeated measurements. Among the most relevant results, it was observed that positive affects were more present in the sample in question. We also found that educators from Kindergarten had less positive affect than others, a factor that may have been influenced by singularities perceived by the participants, for example relating to remuneration, working hours and career plan. No differences were found regarding gender and age group. We argue that further studies on this issue are necessary.
\end{abstract}

Keywords: Positive Psychology, affection, teachers.

\section{Afectos positivos y negativos en profesores de diferentes niveles de enseñanza}

\section{Resumen}

Este trabajo tuvo el objetivo de comparar afectos positivos y negativos de profesores de diversos niveles de enseñanza. Participaron de la investigación 101 profesores - de los cuales 83,2\% ( $n=84)$ eran del sexo feminino, con edad promedio de 39,3 años (SD=9,8). El instrumento utilizado fue la Escala de Afectos Zanon-EAZ. Entre los resultados más relevantes se observó que los afectos positivos estuvieron más presentes en la muestra. También se verificó que los educadores de guardería presentaron menos afectos positivos que los demás profesores. Este factor puede haber sido influenciado por singularidades percibidas por los participantes, relacionadas, por ejemplo, a la remuneración, carga horaria y plan de carrera. No fueron encontradas diferencias en cuanto al sexo, a la edad y a la franja etaria. Se observou la necesidade de realizar más estudios sobre esta temática.

Palabras Clave: Psicología Positiva; afectividad; profesores. 


\section{Introdução}

A Psicologia Positiva propõe uma visão singular de sujeito, priorizando a potencialidade de desenvolvimento de forças e virtudes e o bem-estar nos níveis psicológico, biológico e social (Peterson \& Seligman, 2004). O passado da Psicologia estudou as doenças, o que, de acordo com Peterson e Park (2006) não deve ser abandonado; no entanto, diferentes questões devem ser assumidas. Assim, deve-se buscar compreender o que pode tornar a vida dos indivíduos mais feliz e produtiva bem como identificar e criar talentos (Paludo \& Koller, 2007; Peterson \& Seligman, 2004). Esta abordagem foi criada com o intuito de contribuir com a procura do que faz as pessoas sentirem-se preenchidas e, de fato, felizes, e gerou, além de grande interesse da população leiga, inúmeros investimentos de pesquisa em temas como o otimismo, as emoções positivas, a felicidade e os traços de personalidade saudáveis (Galinha \& Pais-Ribeiro, 2005).

Durante muito tempo os traços negativos relacionados a doenças foram foco de estudo da Psicologia, mas dentro desse novo movimento da Psicologia Positiva o modelo de doença passa a coexistir com a busca pelo bem-estar, pela satisfação com a vida, pela felicidade, por outros aspectos positivos da vida e pela busca da saúde em geral (Ferreira, 2012). As intervenções devem tentar cultivar e sustentar uma boa vida para os homens (Peterson \& Park, 2006).

Para se referir às experiências afetivas vivenciadas pelas pessoas a Psicologia Positiva tem se utilizado do conceito de bem-estar subjetivo (BES) (Nunes, Hutz, \& Giacomoni, 2009). Segundo Albuquerque, Noriega, Coelho, Neves e Martins (2006), estudos referentes ao BES são extremamente relevantes, pois podem auxiliar no planejamento de políticas públicas e fornecer índices de bem-estar e desenvolvimento humano.

Os estudos sobre BES, como afirmam Gouveia, Fonseca, Lins e Gouveia (2008), verificam o modo como as pessoas pensam e sentem a vida. De acordo com Diener (1984), Diener e Suh (1997), Diener, Oishi e Lucas (2003) e Diener, Suh, Lucas e Smith (1999), o BES é composto por elementos cognitivos e emocionais. $\mathrm{O}$ aspecto cognitivo refere-se à avaliação da satisfação com a vida, enquanto o aspecto emocional inclui o afeto manifestado em cada momento, considerando a intensidade e duração das emoções positivas e negativas. O BES é, então, composto pela satisfação com a vida e pelos afetos - positivos e negativos (Diener e cols., 1999). Snyder e Lopez (2009) caracterizaram o afeto como um avaliador dos eventos ocorridos, baseado nas sensações vivenciadas tanto de prazer quanto de dor. Lyubomirsky, King e Diener (2005) apontaram que os afetos contemplam a frequência e intensidade com que os indivíduos experimentam as emoções.

Para Diener (1995), os afetos estão organizados em positivos e negativos, conforme já referido anteriormente, sendo que o negativo é um estado passageiro que engloba emoções desagradáveis, caracterizando-se como comportamentos ansiosos, aborrecidos, pessimistas, aflitivos e angustiantes; e o positivo caracteriza-se por um estado de alerta e atividade, sendo um sentimento passageiro de prazer. Lyubomirsky e cols. (2005) indicaram que altos níveis de afetos positivos relacionam-se com confiança, otimismo, autoeficácia, comportamentos pró-sociais, sociabilidade, energia, originalidade, flexibilidade, imunidade, etc., e com a vivência frequente de situações de prazer. Por outro lado, indivíduos que possuem altos níveis de afetos negativos vivem constantemente situações ligadas ao desprazer. Pessoas com predominância de afetos positivos tendem a ser bem-sucedidas e realizadas em diversos âmbitos de suas vidas. A felicidade mostra-se mais baseada na constância com que as pessoas experimentam as emoções positivas do que na intensidade com a qual estas são vivenciadas, indicando a importância da frequência em face da intensidade das emoções (Lyubomirsky e cols., 2005).

Snyder e Lopez (2009), em defesa à criação desse movimento da Psicologia, refletiram que as informações negativas tendem a exercer mais poder nas pessoas do que as positivas e, desse modo, o bom é menos impactante do que o ruim. Segundo os autores, é necessária a promoção do "bom", em vez de permitir que o ruim controle as experiências dos indivíduos.

Orientados por essa perspectiva, alguns estudos já foram efetivados no Brasil com vistas à construção e validação de instrumentos para avaliação dos construtos definidos pela Psicologia Positiva, com a finalidade de relacioná-los a variáveis externas como sexo e idade, por exemplo. Entre eles encontra-se o de Albuquerque e Trócolli (2004), que, ao considerarem a deficiência de escalas que avaliam o construto dos afetos no Brasil, apresentaram o processo de construção da Escala de Bem-Estar Subjetivo, a qual avalia a satisfação com a vida, afetos positivos e negativos. A pesquisa contou com 795 participantes, com idade média de 35,6 anos, sendo $74 \%$ da amostra do sexo masculino. Além de evidenciarem a validade do instrumento, os autores sugeriram que a população brasileira parece ter mais facilidade em concordar com itens que avaliam os afetos positivos do que com aqueles que avaliam os negativos, o que consideram um produto da cultura; mas segundo os mesmos autores, é necessário fazer um estudo transcultural para confirmar ou não essa suposição.

Com vistas a construir e validar uma escala de afetos positivos e negativos para crianças, Giacomoni e Hutz (2006) fizeram um estudo em que contaram com a participação de 661 crianças, das quais $52,2 \%$ eram do sexo masculino. As crianças eram provenientes de escolas públicas e privadas de Porto Alegre e suas idades variavam entre sete e doze anos. Os resultados apontaram menores índices de afetos positivos para as meninas do que para os meninos. Com relação à idade, os autores perceberam uma baixa nos afetos positivos à medida que aumentavam as faixas etárias.

Fonseca, Chaves e Gouveia (2006) relacionaram valores humanos e BES e avaliaram 313 professores do Ensino Fundamental da Paraíba. Dentre os achados, os autores encontraram que, mesmo parecendo gozar de afetos positivos, os professores tiverem altas pontuações em depressão. Na opinião dos autores, tal fato se deve a uma 
deterioração no bem-estar dos professores investigados, explicada possivelmente pelo ambiente que eles enfrentam, muitas vezes vivenciando estados de tristeza, desânimo, solidão e melancolia, sintomas típicos da depressão.

Com o objetivo de validar a Escala de Bem-Estar Afetivo no Trabalho (JAWS) para a população brasileira, Gouveia e cols. (2008) fizeram um estudo em que contaram com a participação de 298 trabalhadores, com idade média de 25,8 anos e (em sua maioria (76,8\%), mulheres (76,8\%). De acordo com os autores, os resultados indicaram não haver diferença nos afetos, quando consideradas características referentes a gênero e idade.

Para buscar associações entre o BES e o modelo dos cinco grades fatores avaliado pela Bateria Fatorial de Personalidade, Nunes e cols. (2009) realizaram um estudo com 357 estudantes. Os autores encontraram resultados que sugeriram que a personalidade é um fator relevante na explicação do BES. Além disso, não foram observadas diferenças significativas no BES, principalmente quanto ao sexo.

A investigação das relações entre BES, reflexão, ruminação, neuroticismo e sexo foi objetivo da pesquisa de Hutz e Zanon (2009). O estudo contou com a participação de aproximadamente 361 estudantes universitários de ambos os sexos, com idade média de 19,9 anos, sendo $48,5 \%$ dles do sexo feminino. A ruminação mostrou-se um possível preditor do afeto negativo. Fatores como ansiedade, desajustamento psicossocial, depressão e vulnerabilidade também pareceram se relacionar com o afeto negativo. Os autores indicaram que a reflexão tende a ser mais presente nos indivíduos que apresentam altos níveis de afetos positivos. Indicaram também que quanto mais o indivíduo é ruminativo, menos positiva parece ser sua autopercepção. Adicionalmente, foi encontrada diferença significativa quanto ao sexo dos participantes para o afeto negativo, em que as mulheres apresentaram um índice superior $(M=45,7 ; D P=12,0)$ ao encontrado nos homens $(M=43,00 ; D P=10,1)$.

Zanon, Bastianello, Pacico e Hutz (2013) avaliaram 853 estudantes universitários, com idade média de 21 anos, dos quais $57 \%$ eram do sexo feminino. O estudo foi feito com o objetivo de construir a Escala de Afetos Zanon (EAZ). Os resultados apontaram relação entre os fatores insatisfação com a vida, desesperança quanto ao futuro e baixa autoestima e aspectos relacionados a altos níveis de afetos negativos. Quanto ao sexo, os afetos negativos foram mais presentes nas mulheres em comparação com os afetos positivos, sugerindo que elas ficam mais irritadas, preocupadas e com raiva do que os homens, conforme destacaram os autores.

Tendo em vista Danna e Griffin (1999), que apontaram que não há como separar a vida profissional da vida pessoal do trabalhador, e considerando o que é apresentado por Albuquerque e cols. (2006), ao salientar o uso da Psicologia Positiva como auxílio no planejamento de políticas públicas, haja vista a grande importância da educação como uma delas, justifica-se o objetivo do presente estudo. Em que pese ao fato de estudos terem sido realizados no Brasil com o intuito de mensurar os afetos, parecem ser necessárias outras investigações. O presente estudo objetivou identificar os afetos positivos e negativos de professores e comparar as pontuações de acordo com nível de ensino em que atuam. Pretendeu-se também verificar possíveis diferenças entre os participantes quanto à idade e ao sexo e quanto à autoavaliação dos professores.

\section{Método}

\section{Participantes}

O presente estudo contou com 101 professores de diferentes níveis de ensino. Em relação ao sexo, 83,2\% ( $n=84)$ eram mulheres, sendo apenas $16,8 \%(n=17)$ dos participantes do sexo masculino. A idade variou de 23 a 68 anos, com média de 39,3 anos ( $D P=9,8)$. A amostra foi composta por 18 educadores de Creche, 23 professores de Educação Infantil, 22 de Ensino Fundamental, 20 de Ensino Médio e 18 do Ensino Superior. A coleta foi realizada em sete instituições, das quais cinco eram da rede pública e duas da rede privada, localizadas em cidades da região de Campinas/SP.

Quanto ao perfil profissional dos participantes, $70,2 \%$ tinham mais de uma graduação em nível superior, 19,8\% tinham ao menos uma graduação em nível superior e 9,9\% indicaram a conclusão do Magistério. Mais da metade da amostra (54,4\%) tinha pós-graduação. A média de tempo de formado dos profissionais foi de 14,4 anos ( $D P=9,8)$, sendo que um ano foi o tempo mínimo, e 47, o máximo. Verificou-se que o tempo de exercício da docência dos participantes similar ao tempo de formado, apresentando uma correlação positiva e significativa $(r=0,84 ; p=0,000)$, de modo que a maioria dos profissionais da amostra começou a lecionar logo após a graduação.

\section{Instrumento}

Foi utilizada a Escala de Afetos Zanon (EAZ, Zanon e cols., 2013), que busca mensurar os níveis de afetos positivos e negativos presentes nos indivíduos. Esta escala contém vinte sentenças que descrevem sentimentos e emoções passadas e presentes, dos quais dez são referentes aos afetos positivos e dez aos negativos, em uma escala tipo Likert. Os participantes devem se avaliar de 1 a 5 , de acordo com quanto cada sentença os descreve.

A EAZ, em seu estudo de validade, foi comparada com outro instrumento que também avaliava os afetos e com outros que mediam construtos relacionados, quais sejam, satisfação de vida, esperança, orientação da vida e autoestima. Quanto às evidências de validade, o EAZ apresentou altas correlações com o PANAS ( $r=0,73$ para afetos positivos, $r=0,74$ para negativos), o que significa que de fato os dois instrumentos avaliam o mesmo construto. A partir dos demais instrumentos utilizados, os autores verificaram 
que há correlações positivas entre afeto positivo e satisfação com a vida, otimismo, esperança e autoestima, sendo que com essas variáveis o afeto negativo correlacionou-se de modo negativo ou moderado. O EAZ possui um estudo de precisão para a população brasileira, no qual participaram cerca de 800 estudantes universitários de duas cidades do Rio Grande do Sul, com idade média de 21 anos e $57 \%$ da amostra pertencente ao sexo feminino, como descrito anteriormente.

\section{Procedimentos}

Após a autorização de cada escola para a pesquisa, o projeto foi encaminhado ao comitê de Ética em pesquisa de uma instituição de ensino superior do Interior do Estado de São Paulo. Foram colhidas autorizações dos participantes por meio da assinatura do Termo de Consentimento Livre e Esclarecido, que esclarece a forma de participação, orientando a respeito dos objetivos do estudo. Os instrumentos foram aplicados individual e coletivamente, de acordo com a disponibilidade do participante no ambiente escolar. A aplicação iniciou-se com o rapport, e em seguida foi entregue o instrumento e fornecida a orientação para o preenchimento do questionário. O processo de aplicação teve duração de aproximadamente 30 minutos.
Em conjunto com a EAZ foi utilizado também um questionário sociodemográfico com questões que visam obter dados referentes à caracterização dos participantes, formação e prática dos profissionais, como idade, formação superior, nível de ensino em que leciona, especializações/ pós-graduações que cursou, tempo desde que está formado, tempo de exercício da docência, número de instituições em que trabalha, motivo de sua escolha profissional, se o participante gostaria de mudar de profissão e no caso, que profissão escolheria, e a nota que se atribui quanto ao uso da afetividade em sua prática profissional. Para o presente estudo, algumas informações foram selecionadas para as análises.

\section{Resultados}

O presente estudo objetivou comparar afetos positivos e negativos em professores dos diversos níveis de ensino (Creche, Educação Infantil, Ensino Fundamental, Ensino Médio e Ensino Universitário). Deste modo, serão expostos os resultados obtidos pelas estatísticas descritivas e inferenciais. Os afetos positivos e negativos encontrados estão representados na tabela 1 .

Tabela 1. Estatísticas Descritivas dos Afetos Positivos e Negativos ( $n=101)$.

\begin{tabular}{|c|c|c|c|c|c|c|}
\hline & & Creche & $\begin{array}{l}\text { Educação } \\
\text { Infantil }\end{array}$ & $\begin{array}{l}\text { Ensino } \\
\text { Fundamental }\end{array}$ & Ensino Médio & $\begin{array}{l}\text { Ensino } \\
\text { Superior }\end{array}$ \\
\hline & Média & 36,88 & 43,90 & 44,41 & 44,35 & 43,50 \\
\hline AFETOS & DP & 9,42 & 4,92 & 4,50 & 4,17 & 2,57 \\
\hline \multirow[t]{3}{*}{ POSITIVOS } & Mínimo & 17 & 33 & 33 & 37 & 39 \\
\hline & Máximo & 48 & 50 & 50 & 50 & 48 \\
\hline & Média & 23,53 & 22,39 & 24,74 & 24,40 & 21,72 \\
\hline \multirow{3}{*}{$\begin{array}{l}\text { AFETOS } \\
\text { NEGATIVOS }\end{array}$} & DP & 6,03 & 6,59 & 5,68 & 5,07 & 5,72 \\
\hline & Mínimo & 15 & 10 & 13 & 14 & 13 \\
\hline & Máximo & 35 & 39 & 32 & 32 & 31 \\
\hline
\end{tabular}

Tabela 2. Análise de variância ANOVA pelo coeficiente de Esfericidade Considerada.

\begin{tabular}{lllllll}
\hline Fonte de variância & $S Q$ & $g l$ & $M Q$ & $F$ & $p$ & $E_{t a}^{2}$ \\
\hline Afetos & 17326,931 & 1 & 17326,931 & 486,278 & 0,001 & 0,844 \\
Afetos*Nível de ensino & 412,220 & 4 & 103,055 & 2,892 & 0,027 & 0,114 \\
\hline
\end{tabular}

$S Q=$ soma dos quadrados; gl=graus de liberdade; $M Q=$ média dos quadrados. 
Figura 1. Médias marginais estimadas obtidas nos afetos da EAZ em função do nível de ensino.

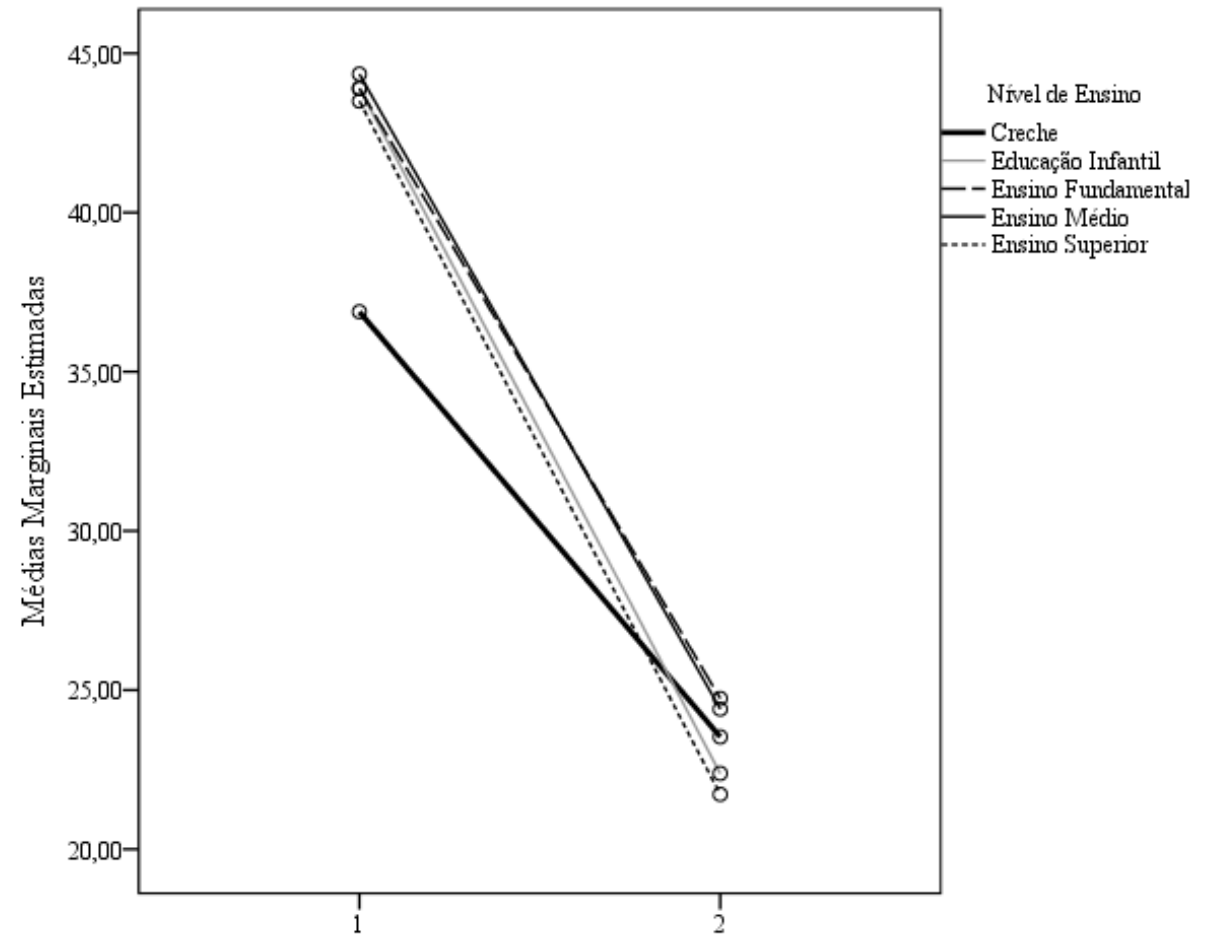

1= Afetos Positivos; 2=Afetos Negativos.

Conforme a tabela 1 , os participantes apresentaram maior frequência de afetos positivos em todos os níveis de ensino, com destaque para a proximidade das médias, excetuando-se apenas os professores de Creche. Por sua vez, quanto aos afetos negativos, além de médias menores, observou-se baixa amplitude na variação entre os níveis.

Para responder ao objetivo proposto, de comparar afetos positivos e negativos de professores dos diversos níveis de ensino, o método estatístico adotado corresponde a uma aplicação específica da ANOVA, apresentada como Análise de Perfis de Medidas Repetidas (Tabachinick \& Fidell, 2007). Tal método analisa, por meio do modelo linear geral (general linear model), se o perfil encontrado nas médias obtidas em cada escala (variáveis dependentes) é distinto para cada nível de ensino (variável independente). Os resultados da ANOVA são apresentados na tabela 2.

Pela análise da tabela 2 se observou que as diferenças encontradas foram significativas tanto intra-afetos quanto na relação entre afetos e nível de ensino. Além disso, também se destaca que $84,4 \%$ da variância dos perfis são explicados pela escala de afetos, e quando associada à variável nível de ensino, há explicação de 11,4\%. Considerando a significância dos coeficientes de variância, confirmando a diferença, a figura 1 apresenta os perfis de cada nível de ensino, representados pelas médias obtidas nas duas escalas de afetos (afetos positivos e afetos negativos).
Pela análise da figura 1 , verificou-se que os educadores, nos diversos níveis de ensino, diferenciam-se quanto à presença dos afetos, visto que os de Creche obtiveram significativamente menor índice de afetos positivos, distanciando-se dos educadores dos demais níveis; mas quanto aos afetos negativos, os professores tendem a apresentar médias próximas e mais baixas, embora diferentes significativamente. Destaque é dado para os perfis muito semeIhantes, que foram apresentados por professores do Ensino Fundamental e Médio.

Outras variáveis também foram investigadas quanto à existência de diferenças significativas em cada categoria. Para esse fim, as pontuações obtidas nos afetos em relação ao sexo foram comparadas, sendo utilizado o teste $t$ de Student. Foi possível verificar que na amostra em questão não houve diferenças significativas entre os sexos quanto aos afetos positivos $[t(101)=0,536 ; p=0,593]$ e negativos $[t(101)=1,338 ; p=0,184]$. Para verificar a existência ou não de diferenças em relação à idade dos participantes, as pontuações obtidas nos afetos foram submetidas à ANOVA, mas não foram encontradas diferenças significativas na escala de afetos positivos $[F(34,63)=0,645 ; p=0,917]$ nem na de afetos negativos $[F(34,62)=0,767 ; p=0,798]$.

Diante desse resultado, julgou-se pertinente verificar se com o agrupamento das idades em faixas etárias as pontuações nos afetos variariam ou não. Os participantes foram 
Tabela 3. Diferença de média entre afetos e nota média $(M=8,47)$ que os participantes se atribuíram.

\begin{tabular}{|c|c|c|c|c|c|c|}
\hline & Nota & $\mathrm{N}$ & Média & $\begin{array}{l}\text { D e s v i o } \\
\text { Padrão }\end{array}$ & $t$ & $p$ \\
\hline Afetos & $\begin{array}{l}\text { Abaixo da } \\
\text { média }\end{array}$ & 48 & 41,16 & 6,21 & \multirow{2}{*}{$-3,323$} & \multirow[b]{2}{*}{0,001} \\
\hline Positivos & $\begin{array}{l}\text { Acima da } \\
\text { média }\end{array}$ & 38 & 45,00 & 3,38 & & \\
\hline \multirow{2}{*}{$\begin{array}{l}\text { Afetos } \\
\text { Negativos }\end{array}$} & $\begin{array}{l}\text { Abaixo da } \\
\text { média }\end{array}$ & 48 & 23,41 & 5,76 & \multirow{2}{*}{0,613} & \multirow{2}{*}{0,542} \\
\hline & $\begin{array}{l}\text { Acima da } \\
\text { média }\end{array}$ & 37 & 22,64 & 5,68 & & \\
\hline
\end{tabular}

organizados em quatro grupos, de acordo com os quartis, a saber: no quartil 25 foram agrupados aqueles com idade inferior a 32 anos; entre os quartís 25 e 50 , os que tinham entre 33 e 39 anos; entre os quatis 50 e 75 , os que tinham entre 40 e 47 anos; e no quartil 75 , aqueles com idade superior a 48 anos. As análises de variância realizadas foram a ANOVA para os quatro grupos e $t$ de Student para os grupos extremos. Ambas as análises indicaram não haver diferenças significativas para os afetos positivos $[F(3,94)=0,456$; $p=0,714]$ e $[t(50)=0,620 ; p=0,538]$ nem para os afetos negativos $[F(3,93)=1,255 ; p=0,294]$ e $[t(51)=1,498 ; p=0,141]$.

Os participantes foram indagados, por meio de um questionário complementar, quanto à nota que se atribuiriam em relação ao uso da afetividade em sua prática profissional. A menor nota observada foi 5 , e a maior, 10 , sendo que a média foi $8,47(D P=1,20)$. A partir dessas médias, foram comparados os grupos extremos que se deram notas abaixo ou acima desses valores. Os resultados obtidos são apresentados na tabela 3 .

Houve uma diferença nos afetos positivos, sendo que os participantes que se atribuíram nota maior também são aqueles que apresentam mais afetos positivos. No que se refere aos afetos negativos, não foram observadas diferenças significativas. Em síntese, os resultados apontaram que houve predominância dos afetos positivos, sendo que o nível de ensino 'Creche' obteve menor índice destes afetos em comparação aos outros níveis de ensino. Com relação ao sexo, à idade e às faixas etárias, não foram observadas diferenças significativas. Por fim, no que diz respeito à nota atribuída ao uso da afetividade, verificou-se que quanto maior a nota, maior também o índice dos afetos positivos dos participantes.

\section{Discussão}

Os resultados apontaram diferença entre os afetos positivos e negativos, sendo que os positivos foram supe- riores na amostra em questão. Fonseca e cols. (2006) igualmente apontaram essa informação, porém salientaram que, mesmo parecendo desfrutar de afetos positivos e satisfação com a vida, os professores se mostraram inclinados a desenvolver depressão. O resultado encontrado também está em concordância com as informações teóricas trazidas por Albuquerque e Trócolli (2004), pois segundo esse resultado a população brasileira, como consequência cultural, apresenta uma tendência de pontuar mais itens que avaliam os afetos positivos do que que itens que avaliam os negativos.

Fizeram parte da amostra professores de cinco níveis de ensino. A diferença entre os afetos positivos e negativos, no que respeita aos vários níveis, revelou que os positivos estavam menos presentes nos profissionais que se dedicavam à Creche, em relação aos profissionais que atuavam em outros níveis. Não foram encontrados outros estudos que se dedicassem a investigar a mesma amostra, o que dificulta o diálogo com esse dado.

Com relação ao sexo, não foram encontradas diferenças significativas entre os afetos, assim como o verificado em Nunes e cols. (2009) e defendido por Diener (1984), Diener e Suh (1997), Diener e cols. (1999, 2003), porém os afetos tiveram índices distintos dos encontrados em Hutz e Zanon (2009). No último, as mulheres apresentaram um índice superior de afetos negativos quando comparadas aos homens. Em outra pesquisa, esta de Zanon e cols. (2013), os afetos negativos também se mostraram mais presentes no sexo feminino, sugerindo maior irritação, preocupação e raiva entre as mulheres do que entre os homens. Adicionalmente, Giacomoni e Hutz (2006), em seu estudo com crianças, perceberam menores índices de afetos positivos no sexo feminino. Diante disso, é importante verificar a divisão da amostra quanto a esse aspecto, sendo que o sexo masculino não exerceu participação expressiva, até pelas características observadas na profissão, em que há predominância do sexo feminino. $\mathrm{Na}$ amostra em questão, embora esse dado não tenha sido formalmente apresentado, durante a coleta foi observado haver mais participantes 
do sexo masculino lecionando apenas no Ensino Médio e Universitário.

A comparação entre afetos positivos e negativos entre pessoas de diferentes faixas etárias foi um dos objetos de investigação deste estudo. Os resultados revelaram que os afetos negativos estão menos presentes nos participantes mais velhos, diferentemente do que encontraram Giacomoni e Hutz (2006), os quais observaram uma diminuição nos afetos positivos à medida que as crianças envelheciam. Não obstante, tal comparação deve ser feita com cautela, uma vez que o último estudo abordou apenas crianças e jovens. Em outra direção, Gouveia e cols. (2008) não encontraram diferenças nos afetos quando consideradas características referentes a gênero e idade. Quando considerada a nota atribuída ao grau da afetividade verificado em sua prática profissional, os participantes que se atribuíram maior nota também apresentaram mais afetos positivos. Os dados permitem compreender que a autoavaliação favorável está relacionada à presença de afetos positivos, o que está em com formidade com os dados de Lyubomirsky e cols. (2005), os quais apontaram confiança e autoeficácia como características de indivíduos com altos índices de afetos positivos. Este fator parece apontar que quanto mais o professor se percebe satisfeito com o trabalho por ele desenvolvido, mais afetos positivos ele parece ter.

Deste modo, foi verificado que os objetivos propostos foram atingidos, permitindo uma reflexão a respeito dos afetos positivos e negativos. As diferenças apresentadas podem ter sido influenciadas por singularidades observadas na amostra em questão, na qual a remuneração é inferior à dos demais e a carga horária é a maior, se comparada aos profissionais de outros níveis. Os demais profissionais podem dobrar o período de trabalho, devido à carga horária inferior, diferentemente dos profissionais da Creche. Outro fator relevante é a designação de educadores em vez de professores, e também o emprego da nomeação "tia" pelos alunos e seus familiares, aspecto que não necessariamente agrada os profissionais deste nível de ensino. A falta de plano de carreira também é um fator negativo, sendo que os educadores de Creche não podem exercer atividades de coordenação escolar, a não ser mediante concurso. Cabe ressaltar que os professores de outros níveis de ensino podem assumir a função de coordenação, caso sejam convidados. A Creche, assim como a Educação Infantil, também exige uma polivalência na formação e uma relação mais próxima entre o professor e as famílias, que podem ser fatores geradores de afetos negativos.

Diante da maior presença de afetos positivos em professores pós-graduados, pode-se inferir que investir na formação reflete a preocupação do indivíduo em melhorar seu trabalho, denunciando o alto envolvimento entre o profissional e as atividades por ele desenvolvidas. Além disso, parece expressar um autoconceito positivo e confiança deste indivíduo, que busca aprimorar-se em sua formação. Outra hipótese pode estar relacionada com a baixa remuneração desta classe de profissionais, que, investindo em especia- lizações, buscam melhores oportunidades e condições de trabalho.

Vale também ressaltar o que é apontado por Albuquerque e cols. (2006), segundo os quais, estudos dos componentes do BES auxiliam no planejamento de políticas públicas. A este respeito, convém reafirmar as asserções de Peterson e Seligman (2004), de que o movimento da Psicologia Positiva considera que emoções positivas e negativas são autênticas, no entanto, é possível construir rotas para uma vida psicológica mais positiva.

Por outro lado, é importante considerar as limitações da pesquisa, a amostra restrita, e as condições de trabalho dos professores estudados, que provavelmente são diferentes se considerados outro contexto e outra região. A título de agenda de pesquisas, sugere-se que sejam desenvolvidos estudos com amostras maiores e que nestas a distribuição por sexo seja mais igualitária. Ainda, pode-se diferenciar o nível do Ensino Fundamental em suas etapas I e II, verificar se há diferença entre os professores da rede pública e os de escolas privada, entre aqueles com formação presencial aqueles formados pelo sistema de ensino a distância, entre os que têm pós-graduação stricto sensu e os que têm pós graduação lato sensu, entre outras variáveis não contempladas na presente investigação.

\section{Referências}

Albuquerque, A. S., \& Trócolli, B. T. (2004). Desenvolvimento de uma escala de bem estar subjetivo. Psicologia: Teoria e Pesquisa, 20(2), 153-164.

Albuquerque, F. J. B., Noriega, J. A. V., Coelho, J. A. P. M., Neves, M. T. S., \& Martins, C. R. (2006). Valores básicos como preditores do bem-estar subjetivo. PSICO, 37(2), 131-137.

Danna, K., \& Griffin, R. W. (1999). Health and Well-Being in the Workplace: A Review and Synthesis of the Literature. Journal of Management, 25(3), 357-384.

Diener, E. (1984). Subjective Well-Being. Psychological Bulletin, 95(3), 542-575.

Diener, E. (1995). A value based index for measuring national quality of life. Social Indicators Research, 36, 107-127.

Diener, E., \& Suh, E. (1997). Mesuring quality of life: Economic, social, and subjective indicators. Social Indicators Research, 40, 189-216.

Diener, E., Oishi, S., \& Lucas, R. E. (2003). Personality, Culture and Subjective well-being: emocional and cognitive evaluations of life. Annual Review Psychology, 54, 403-425.

Diener, E., Suh, E. M., Lucas, R. E., \& Smith, H. L. (1999). Subjective Well-Being: Three Decades of Progress. Psychological Bulletin, 125(2), 276-302. 
Ferreira, M. C. (2012). Antecedentes individuais do bem-estar no trabalho sob a ótica da Psicologia Positiva. Em M. C. Ferreira \& H. Mendonça (Orgs.), Saúde e bem-estar no trabalho: dimensões individuais e culturais (pp. 157-176). São Paulo: Casa do Psicólogo.

Fonseca, P. N., Chaves, S. S. S., \& Gouveia, V. V. (2006). Professores do ensino fundamental e o bem estar subjetivo: uma explicação baseada em valores. Psico USF, 11(1), 45-52.

Galinha, I. C., \& Pais-Ribeiro, J. L. (2005). Contribuição para o estudo da versão portuguesa da Positive and Negative Affect Schedule (PANAS): I - Abordagem teórica ao conceito de afecto. Análise Psicológica, 2(23), 209-218.

Giacomoni, C. H, \& Hutz, C. S. (2006). Escala de afetos positivo e negativo para crianças: estudos de construção e validação. Psicologia Escolar Educacional, 10(2), 235-245.

Gouveia, V. V., Fonseca, P. N., Lins, S. L. B., Lima, A. V., \& Gouveia, R. S. V. (2008). Escala de bem-estar afetivo no trabalho (Jaws): Evidências de validade fatorial e consistência interna. Psicologia: Reflexão e Crítica, 21(3), 464-473.

Hutz, C. S., \& Zanon, C., (2009). Relações entre bem-estar subjetivo, neuroticismo, ruminação, reflexão e sexo. Revista Interinstitucional de Psicologia, 2(2), 118-127.
Lyubomirsky, S, King, L., \& Diener, E. (2005). The benefits of positive affect: Does hapinees lead to success? Psychological Bulletin, 131(6), 803-855.

Nunes, C. H. S., Hutz, C. S., \& Giacomoni, C. H. (2009). Associação entre o bem estar subjetivo e personalidade no modelo dos cinco grandes fatores. Avaliação Psicológica, 8(1), 99-108.

Paludo, S. S., \& Koller, S. H. (2007). Psicologia Positiva: uma nova abordagem para antigas questões. Paidéia, 17(36), 9-20.

Peterson, C., \& Park, N. (2006). Classification and Measuremente of Character Strengths: implications for Practice. Em P. A. Linley \& S. Joseph (Eds.), Positive psychology in practice (pp. 433-446). Hoboken, NJ: Wiley.

Peterson, C., \& Seligman, M. E. P. (2004). Character Strengths and Virtues - a handbook and classification. Oxford: American Psychological Association, Oxford University Press.

Snyder, C. R., \& Lopez, S. J. (2009). Psicologia Positiva. Porto Alegre: Artmed.

Tabachinick, B. G., \& Fidell, L. S. (2007). Using multivariate statistics (5a ed). New York: Pearson Education, Inc.

Zanon, C., Bastianello, M., Pacico, J. C., \& Hutz, C. S. (2010). Escala de Afetos Zanon. Psico-USF, 18(2), 193-202.

Recebido em : 13/11/2012

Reformulado em: 02/10/2013

Aprovado em: 16/12/2013

\section{Sobre as autoras}

Ana Paula Porto Noronha (ana.noronha@usf.edu.br)

Doutora em Psicologia Ciência e Profissão pela Pontifícia Universidade Católica de Campinas. Docente do Programa de Pós-Graduação Stricto Sensu em Psicologia da Universidade São Francisco. Bolsista Produtividade em Pesquisa do CNPq.

Mariana Palladino Delforno (mapalladino@gmail.com)

Psicóloga graduada pela Universidade São Francisco. Pedagoga graduada pela Universidade Paulista.

Lariana Paula Pinto (paula.lariana@gmail.com)

Mestre e doutoranda em Psicologia pelo Programa de Pós-Graduação Stricto Sensu em Psicologia da Universidade São Francisco. Bolsista CAPES. 